Virginia Commonwealth University vCU Scholars Compass

\title{
Vapor phase nucleation on neutral and charged nanoparticles: Condensation of supersaturated trifluoroethanol on $\mathrm{Mg}$ nanoparticles
}

Victor Abdelsayed

Virginia Commonwealth University

M. Samy El-Shall

Virginia Commonwealth University, selshall@vcu.edu

Follow this and additional works at: http://scholarscompass.vcu.edu/chem_pubs

Part of the Chemistry Commons

Abdelsayed, V., El-Shall, M. S. Vapor phase nucleation on neutral and charged nanoparticles: Condensation of supersaturated trifluoroethanol on Mg nanoparticles. The Journal of Chemical Physics 126, 024706 (2007). Copyright (C) 2007 AIP Publishing LLC.

\section{Downloaded from}

http://scholarscompass.vcu.edu/chem_pubs/61

This Article is brought to you for free and open access by the Dept. of Chemistry at VCU Scholars Compass. It has been accepted for inclusion in Chemistry Publications by an authorized administrator of VCU Scholars Compass. For more information, please contact libcompass@vcu.edu. 


\title{
Vapor phase nucleation on neutral and charged nanoparticles: Condensation of supersaturated trifluoroethanol on $\mathrm{Mg}$ nanoparticles
}

\author{
Victor Abdelsayed and M. Samy El-Shall ${ }^{\text {a }}$ \\ Department of Chemistry, Virginia Commonwealth University, Richmond, Virginia 23284-2006
}

(Received 20 October 2006; accepted 10 November 2006; published online 11 January 2007)

\begin{abstract}
A new technique is described to study the condensation of supersaturated vapors on nanoparticles under well-defined conditions of vapor supersaturation, temperature, and carrier gas pressure. The method is applied to the condensation of supersaturated trifluoroethanol (TFE) vapor on $\mathrm{Mg}$ nanoparticles. The nanoparticles can be activated to act as condensation nuclei at supersaturations significantly lower than those required for homogeneous nucleation. The number of activated nanoparticles increases with increasing the vapor supersaturation. The small difference observed in the number of droplets formed on positively and negatively charged nanoparticles is attributed to the difference in the mobilities of these nanoparticles. Therefore, no significant charge preference is observed for the condensation of TFE vapor on the Mg nanoparticles. (C) 2007 American Institute of Physics. [DOI: 10.1063/1.2404679]
\end{abstract}

\section{INTRODUCTION}

Ion-induced nucleation is a phenomenon of great importance for many scientific, atmospheric, environmental, and industrial applications. ${ }^{1}$ Among these are upper atmospheric chemistry, ${ }^{2-4}$ formation of acid rain and environmental pollutants in the presence of $\mathrm{SO}_{3}^{+}$and $\mathrm{NO}_{2}^{+}$ions,${ }^{5,6}$ the formation of jet contrails, ${ }^{7}$ cosmic rays and condensation of interstellar dust, ${ }^{8}$ and other applications in radiation chemistry, combustion processes, chemical vapor deposition, and plasma processes. ${ }^{9,10}$

The history of ion-induced nucleation research began more than 100 years ago dating back to the original experiments by Wilson and the theoretical formulation by Thomson. ${ }^{11}$ In 1895, Wilson performed his first experiments of making clouds by expansion of moist air, and reported that the water clouds formed in an expansion cloud chamber occurred at lower expansion ratios in the presence of ions than in their absence. ${ }^{11,12} \mathrm{He}$ also observed that nucleation of water vapor occurred on negative ions at lower expansions than on positive ions, thus suggesting that water nucleates preferentially on negatively charged ions (this has been referred to in the nucleation literature as a sign effect in ion-induced nucleation). Wilson received a Nobel Prize in 1920 "for his method of making the paths of electrically charged particles visible by condensation of vapor."12

Because of the strong ion-dipole and ion-induced dipole forces relative to van der Waals dispersion forces, ion nucleation occurs preferentially with respect to homogeneous nucleation. Many other species such as surfaces, foreign materials, and nanoparticles may serve to lower the thermodynamic barrier to nucleation by providing the interface required for nucleation since a surface already exists. In many natural and practical processes the condensation of supersaturated vapors occurs on charged nanoparticles, where both

\footnotetext{
${ }^{\text {a) }}$ Author to whom correspondence should be addressed; FAX: +1 804828 1280. Electronic mail: selshall@hsc.vcu.edu
}

ion-induced and heterogeneous nucleation mechanisms are simultaneously involved. ${ }^{9,13}$ The properties of the preexisting nuclei and the condensing vapor influence the nucleation process. For example, for charged nanoparticles, the size of the particles, the surface properties, the contact angle between the condensing molecules and the particle, as well as the sign and the magnitude of the charge play the most important roles on the thermodynamics and kinetics of the process. The molecular properties of the condensing vapor molecules such as dipole moment and polarizability can also affect the nucleation process. Although the importance of these parameters has been well-recognized, quantitative information on the heterogeneous nucleation of supersaturated vapors on charged nanoparticles under well defined supersaturation and temperatures are rarely available. ${ }^{14-18}$

Most of the recent studies have focused on the application of the condensation nuclei counter (CNC) and the flow cloud chamber (FCC) to investigate the condensation of supersaturated vapors on neutral and charged particles. ${ }^{15-18}$ Despite the practical advantages of the CNC and FCC devices, accurate determination of the degree of the vapor supersaturation at well-defined temperatures is not possible. On the other hand, the thermal diffusion cloud chamber (DCC) allows accurate measurements of both temperature and supersaturation over a wide range of carrier gas pressures. The DCC has been extensively used to study different types of vapor phase nucleation including homomolecular and binary homogeneous nucleation, and photoinduced and ion-induced nucleation. ${ }^{19-23}$ By coupling pulsed laser vaporization of metals to generate charged nanoparticles within the supersaturated vapor in the DCC ${ }^{24}$ it is now possible to measure a time-dependent nucleation rate following the generation of the nanoparticles as a function of vapor supersaturation, carrier gas pressure, nanoparticle concentration as well as the sign of the charged nanoparticles.

In this paper, we demonstrate the application of a new technique to investigate the condensation of supersaturated 


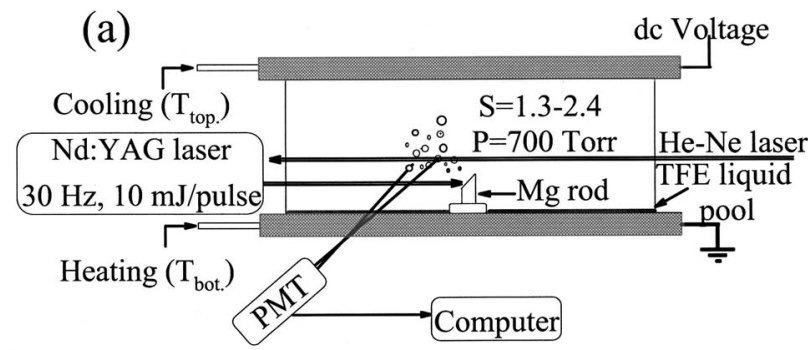

(b)

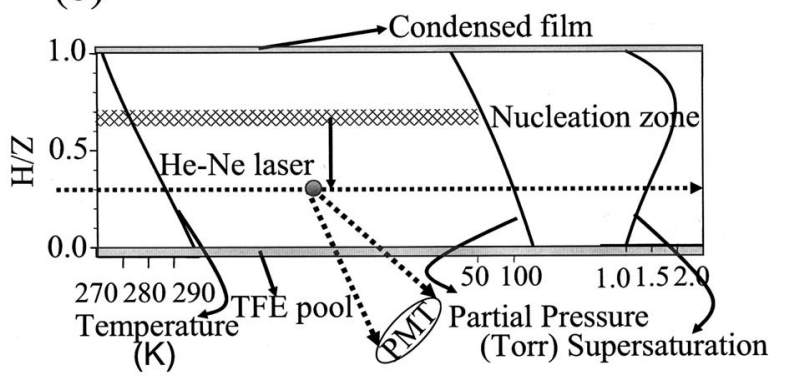

FIG. 1. (a) Experimental setup for the diffusion cloud chamber used for the vapor phase nucleation on $\mathrm{Mg}$ nanoparticles. (b) Temperature, partial pressure, and supersaturation profiles calculated for the observed nucleation of $1-3$ drops $\mathrm{cm}^{-3} \mathrm{~s}^{-1}$ for TFE at $T_{\text {bottom }}=305 \mathrm{~K}, T_{\text {top }}=267 \mathrm{~K}$, and $P_{\text {total }}$ $=700$ Torr.

2,2,2 trifluoroethanol (TFE) vapor on magnesium ( $\mathrm{Mg}$ ) nanoparticles generated by pulsed laser vaporization inside a DCC. The results provide quantitative nucleation data on charged and neutral $\mathrm{Mg}$ nanoparticles under well-defined conditions of vapor supersaturation, temperature, and carrier gas pressure as well as the effects of applied electric field and laser vaporization power. The results provide new insight on the condensation of supersaturated vapors on nanoparticles including the sign effect of the charged nuclei.

\section{EXPERIMENT}

A detailed description of the DCC and principles of its operation are available in the literature, ${ }^{19-23}$ and only a brief description is given here (see Fig. 1). The chamber consists of two aluminum circular plates separated by a glass ring. A magnesium rod (Aldrich, 99.9+\%) of diameter $6.0 \mathrm{~mm}$ and height of $11.5 \mathrm{~mm}$ was placed on the center of the bottom plate. A shallow pool of TFE liquid $(75 \mathrm{~mL}$, Aldrich NMR grade, $99.5+\%$ ) was introduced to the bottom plate. The $\mathrm{Mg}$ rod was isolated from the TFE liquid by a Teflon block. The total pressure inside the chamber was adjusted to about 700 Torr, at room temperature, by introducing an inert, noncondensable gas (He, 99.999\%). A temperature difference was set between the top and the bottom plates, to control the vapor supersaturation $(S)$. The chamber profiles showing supersaturation ratio, partial pressure, and temperature as a function of reduced height (obtained by solving the mass and heat transfer equations ${ }^{19}$ ) are shown in Fig. 1(b). In these experiments, the vapor supersaturation of TFE was adjusted below that required for homogeneous nucleation. A single laser pulse $\left(1.6 \times 10^{8} \mathrm{~W} / \mathrm{cm}^{2}\right)$ was fired from a Quanta ray Nd:yttrium aluminum garnet (YAG) laser (532 nm, $2 \mathrm{~ns}$

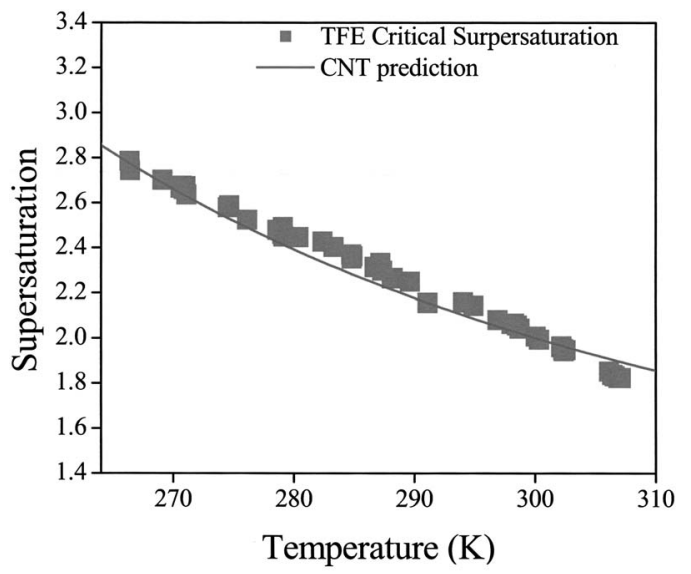

FIG. 2. Critical supersaturation vs temperature for 2,2,2-trifluoroethanol: the solid line represents the classical nucleation theory $(\mathrm{CNT})$ prediction.

pulse width) focused on the $\mathrm{Mg}$ target. The $\mathrm{He}-\mathrm{Ne}$ laser beam was set above that of the Nd:YAG laser to detect droplets of TFE-coated Mg nanoparticles. The nucleating droplets were counted by observing the forward scattering of light from the droplets falling through a horizontal He-Ne laser beam traversing the chamber using a photomultiplier connected to counting electronics. The number of droplets was counted within a well-defined volume. Each data point was repeated at least three times.

\section{RESULTS AND DISCUSSION}

We measured the critical supersaturations $\left(S_{c}\right)$ of pure TFE within the temperature range of 266-307 K (the supersaturation required for a homogeneous nucleation rate of $1-2$ drops $/ \mathrm{cm}^{3} / \mathrm{s}$ ). Figure 2 shows the temperature dependence of the critical supersaturation for TFE along with the predictions of the classical nucleation theory. A good agreement was observed between the experimental results and the theory over the entire temperature range.

The condensation experiments on the Mg nanoparticles were performed with the supersaturation of the TFE vapor significantly below the value required for homogeneous nucleation (for example, $S_{c}=2.6$ at $T=269.4 \mathrm{~K}$ ). As a result, no droplets were observed in the absence of $\mathrm{Mg}$ nanoparticles. Furthermore, when the laser pulse was fired on the $\mathrm{Mg}$ target no droplets were observed if the TFE supersaturation was below $50 \%-70 \%$ of the critical supersaturation at any given temperature. This indicates that the $\mathrm{Mg}$ nanoparticles generated by laser vaporization could not be activated for condensation of the TFE vapor at such low supersaturations. It was also important to exclude the possibility of photoinduced nucleation resulting from the interaction of the $532 \mathrm{~nm}$ laser pulse with the supersaturated TFE vapor. When the laser pulse was fired inside the chamber without hitting the $\mathrm{Mg}$ target, no nucleation events were observed even at supersaturations as high as $95 \% S_{c}$ indicating the absence of photoinduced nucleation under the experimental conditions of the $\mathrm{Mg}$ nanoparticle experiments. It should be noted that under the laser power used in the current experiments a single laser pulse generates about $10^{13}-10^{14}$ metal atoms including about $10^{5}-10^{6}$ atomic ions. ${ }^{25}$ These atoms immedi- 


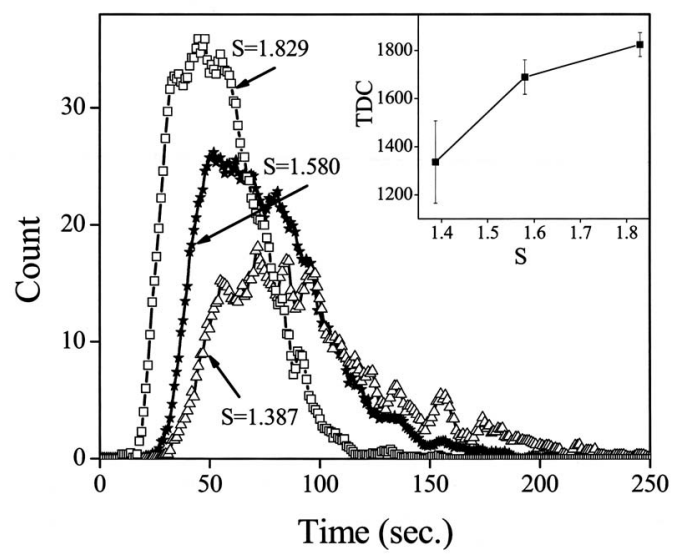

FIG. 3. Time profiles showing the number of TFE droplets condensed on $\mathrm{Mg}$ nanoparticles after a single laser pulse $\left(1.6 \times 10^{8} \mathrm{~W} / \mathrm{cm}^{2}\right)$ at different supersaturations. Inset shows the total number of droplets (integrated time profile) as a function of supersaturation. These experiments were performed at $T_{\max }=281.4 \mathrm{~K}, P=674.7$ Torr, and $E=5.54 \times 10^{3} \mathrm{~V} / \mathrm{m}$.

ately condense to form neutral nanoparticles with a small fraction of charged ones. The nanoparticles diffuse through the DCC where they may become activated in the nucleation zone $(\sim 0.7$ reduced height of the DCC) thus resulting in the condensation of the supersaturated TFE vapor. The activation process depends on the size and charge of the $\mathrm{Mg}$ nanoparticles as well as the degree of the supersaturation of the TFE vapor. In the following sections, the effects of vapor supersaturation, carrier gas pressure, vaporization laser power, and applied electric field are presented and discussed.

\section{A. Effect of vapor supersaturation}

Figure 3 displays the nucleation time profiles following a single laser vaporization pulse on the $\mathrm{Mg}$ target at various TFE supersaturations (1.387-1.829) at a total pressure of 675 Torr. In these experiments, an electric field of 5.54 $\times 10^{3} \mathrm{~V} / \mathrm{m}$ was applied between the chamber plates. It is clear that the total number of droplets (proportional to the rate of nucleation) decreases by decreasing the supersaturation of the TFE vapor. The nucleation peak is also shifted to longer times as the supersaturation ratio of the TFE vapor is decreased. At TFE supersaturations of 1.829, 1.580, and 1.387, the nucleation peak occurs at about 45, 52, and $72 \mathrm{~s}$ from the laser pulse, respectively.

From the above results, it is evident that at higher supersaturations most of the $\mathrm{Mg}$ nanoparticles are activated and the nucleation rate reaches its maximum at early times. On the other hand, at a low supersaturation $(S=1.387)$ only large $\mathrm{Mg}$ nanoparticles can be activated, and since these particles diffuse slowly the nucleation rate reaches its maximum at a longer time (72 s). This behavior is a direct result of the dependence of the critical size (nucleus) on supersaturation according to the classical nucleation theory which is given by $^{1}$

$$
n^{*}=\left[32 \pi \sigma^{3} v^{2}\right] / 3\left(k_{B} T \ln S\right)^{3},
$$

where $\sigma$ is the surface tension of the liquid, $v$ is the molecular volume, $k_{B}$ is Boltzmann's constant, and $S$ is the vapor supersaturation ratio. Equation (1) can be written in terms of the Kelvin diameter using molar quantities as

$$
d^{*}=4 M \sigma / \rho R T(\ln S)
$$

where $M$ is the molecular weight of the condensing vapor molecules. Particles smaller than the Kelvin diameter are not activated and eventually condense on the chamber plates. ${ }^{1}$ The Kelvin diameters calculated using Eq. (2) for the supersaturated TFE vapor at $S=1.829,1.580$, and 1.387 are 4.5, 5.9 , and $8.3 \mathrm{~nm}$, respectively. Therefore, it is clear that by increasing the supersaturation, the Kelvin diameter decreases, and more particles should be activated and grow to macroscopic liquid droplets.

Figure 3 also shows that the induction time (the time required for the onset of nucleation from the laser pulse) increases with decreasing the vapor supersaturation. This is also a direct manifestation of the slow diffusion of the large $\mathrm{Mg}$ nanoparticles that act as nuclei for the condensation of TFE vapor at low supersaturations. These results are in good agreement with the results of Mavliev et al., ${ }^{15}$ Chen et al., ${ }^{17,18}$ and Rosenorn et al. $^{26}$ where the critical supersaturation of the condensing vapor required for activating the nucleating particles was found to decrease with increasing the particle diameter. Similarly, the work of Seto et al. ${ }^{27}$ on the condensation of dibutyl phthalate vapor induced by seed ions generated by electrospray ionization showed a significant reduction in the critical supersaturation with increasing the size of the seed ions.

\section{B. Effect of carrier gas pressure}

The effect of carrier gas pressure on the condensation of supersaturated TFE vapor $(S=1.823)$ on the $\mathrm{Mg}$ nanoparticles was studied in the presence of an electric field of $1.54 \times 10^{2} \mathrm{~V} / \mathrm{m}$. Figure $4(\mathrm{a})$ displays the nucleation time profiles at three different pressures (328, 620, and 934 Torr). It is clear that the width of the nucleation time profile and the total number of droplets increase with increasing the carrier gas pressure. These results can be explained by the laser vaporization process which depends mainly on the ambient gas used and its pressure. ${ }^{25,28}$ For example, the expansion of the laser vaporization plume is more confined at higher pressures and with heavier carrier gases. ${ }^{28,29}$ Therefore, at lower helium pressures, it is expected that most of the small $\mathrm{Mg}$ nanoparticles diffuse quickly through the nucleation zone before they can be activated to condense the supersaturated TFE vapor. However, at higher pressures, the plume is confined in space and larger $\mathrm{Mg}$ nanoparticles can be formed. These nanoparticles, which diffuse slowly, are more likely to be activated in the nucleation zone and therefore, at higher helium pressure the total number of droplets and their residence times increase. The inset in Fig. 4(a) shows a nearly linear relationship between the number of droplets detected and the total pressure in the DCC.

Figure 4(b) displays the effect of carrier gas pressures in the presence of higher electric field $\left(5.54 \times 10^{3} \mathrm{~V} / \mathrm{m}\right)$ and higher vapor supersaturation as compared to the field and supersaturation used in Fig. 4(a). As expected, the nucleation 

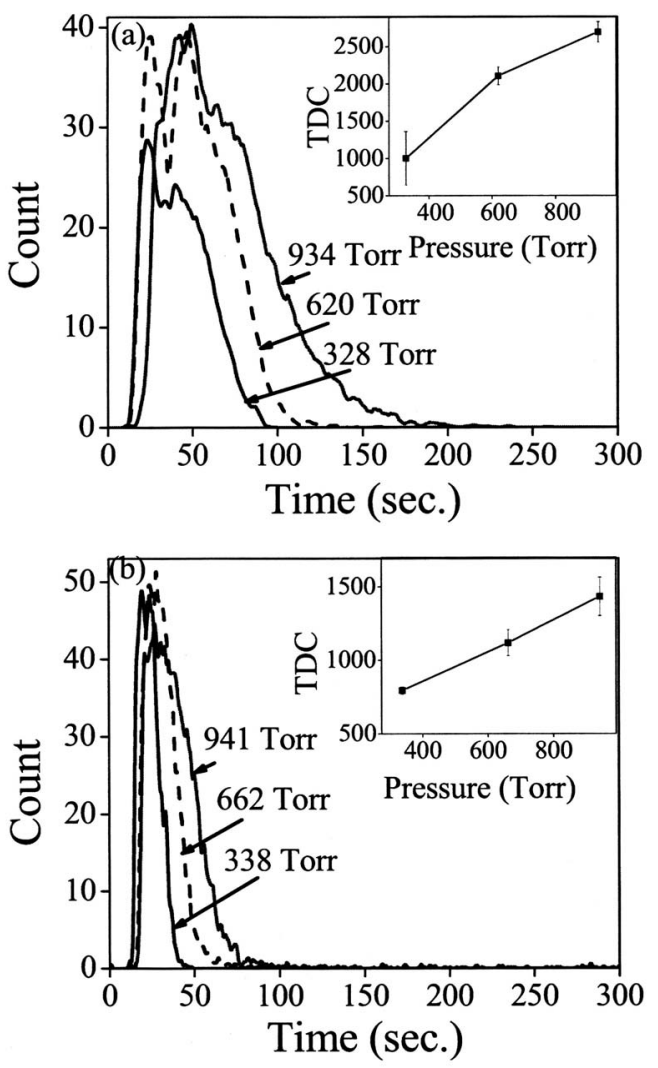

FIG. 4. (a) Time profiles showing the number of TFE droplets condensed on $\mathrm{Mg}$ nanoparticles at $S_{\max }=1.823, T_{\max }=277.6 \mathrm{~K}$, and $E=1.54 \times 10^{2} \mathrm{~V} / \mathrm{m}$ after a single laser pulse at different total pressures. Inset shows the total number of droplets as a function of pressure. (b) Time profiles showing the number of TFE droplets condensed on $\mathrm{Mg}$ nanoparticles at $S_{\max }=2.50$, $T_{\max }=271.6 \mathrm{~K}$, and $E=5.54 \times 10^{3} \mathrm{~V} / \mathrm{m}$ after a single laser pulse $(1.6$ $\left.\times 10^{8} \mathrm{~W} / \mathrm{cm}^{2}\right)$ at different total pressures. Inset shows the total number of droplets as a function of pressure.

time profiles are narrower at higher electric field [Fig. 4(b)] but the trend of increasing the number of droplets with increasing the carrier gas pressure is similar to the trend observed at a lower field [Fig. 4(a)].

\section{Effect of laser power}

All the experiments discussed so far were carried out using a constant laser power density of $1.6 \times 10^{8} \mathrm{~W} / \mathrm{cm}^{2}$. We investigated the effect of laser power at constant total pressure $(P=665.2$ Torr $)$, supersaturation $(S=1.825)$, and small electric field $\left(1.54 \times 10^{2} \mathrm{~V} / \mathrm{m}\right)$. The nucleation time profiles measured following a single laser pulse at different power densities are displayed in Fig. 5. It is clear that as the laser power density increases, the number of activated Mg nanoparticles decreases and the induction time increases. Two important factors could control the observed effect: the mass concentration $(\mathrm{MC})$, and the number concentration $(\mathrm{NC})$ of the nanoparticles generated following the laser pulse. With high laser power density, more material (MC) is vaporized and as a result, larger particles are formed which take a longer time to diffuse to the nucleation zone. Furthermore, heavy particles could be ejected directly from the target surface instead of atomic species. ${ }^{28}$ These heavy particles will most likely fall into the liquid pool, and therefore will not

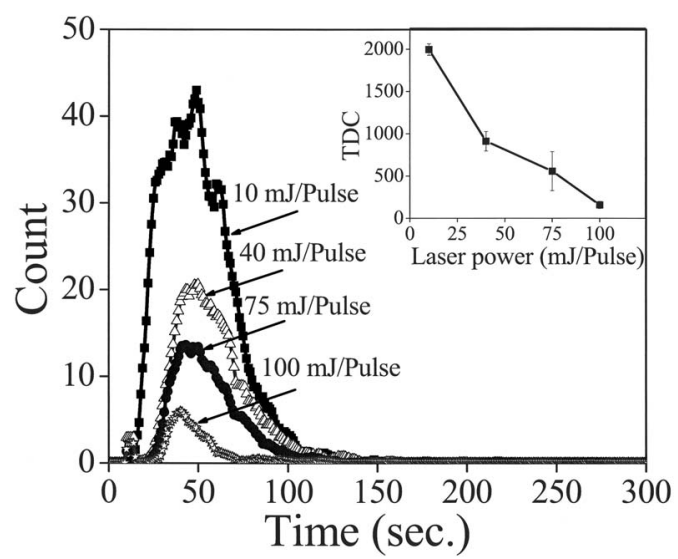

FIG. 5. Time profiles showing the number of TFE droplets $\left(S_{\max }=1.825\right.$, $T_{\max }=277.8 \mathrm{~K}, P=665.2$ Torr, and $E=1.5 \times 10^{2} \mathrm{~V} / \mathrm{m}$ ) after a single laser pulse of different powers. Inset shows the total number of droplets as a function of laser power used for $\mathrm{Mg}$ vaporization.

contribute to the condensation of the TFE vapor. This explains the decrease in the number of activated nanoparticles and the longer induction period at high laser power density. On the other hand, the $\mathrm{NC}$ is expected to increase at low laser powers since most of the evaporated material consists of atomic species which immediately condense to form small nanoparticles. Molecular dynamics simulations of laser ablation of organic solids have found that, below certain laser fluence, primarily single molecules are desorbed while above this threshold, a collective ejection or ablation takes place in which large molecular clusters form a significant portion of the ejected plume. ${ }^{29}$ Additionally, as the laser power increases, the number of ions generated decreases due to the increased recombination probability of the ions with the ejected electrons. ${ }^{30}$ This means that at low power density a large number of charged particles are generated that could diffuse quickly to the nucleation zone to act as nuclei for the condensation of TFE vapor thus resulting in a large number of detectable droplets. The overall trend, shown in the inset of Fig. 5, indicates that the integrated number of droplets falls sharply with increasing the laser power density.

\section{Effect of applied electric field}

Since laser vaporization of the $\mathrm{Mg}$ target generates a fraction of atomic ions, ${ }^{25}$ it is expected to see a significant effect on the nucleation profiles by applying an electric field between the chamber plates. Figure 6 displays the nucleation time profiles in the absence and presence of different electric fields $\left(0.0- \pm 15.38 \times 10^{3} \mathrm{~V} / \mathrm{m}\right)$ at a constant TFE supersaturation ratio of 2.39 and a total pressure of 644 Torr.

It is clear from the results of Fig. 6 that the time width of the nucleation cloud decreases as the applied electric field is increased. For example, the nucleation cloud formed by $\mathrm{Mg}$ nanoparticles at supersaturation of 2.39 with no applied field lasts for more than $50 \mathrm{~s}$ [Fig. 6(a)] while when an electric field of $15.38 \times 10^{3} \mathrm{~V} / \mathrm{m}$ is applied at the same supersaturation of 2.39, the nucleation cloud lasts only for about $30 \mathrm{~s}$ (not shown). The nucleation time profiles displayed in Fig. 6(b) obtained at a supersaturation of 1.819 show that the width and intensity decrease as the applied field increases. 

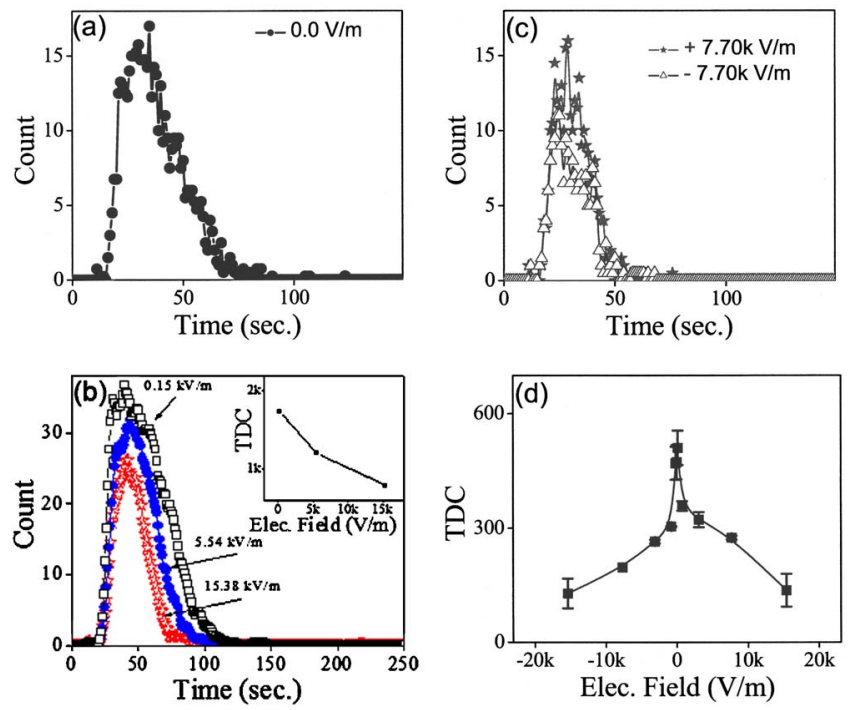

FIG. 6. (a) Nucleation time profile showing the number of TFE droplets $\left(S_{\max }=2.39, T_{\max }=269.0 \mathrm{~K}\right.$, and $P=644.2$ Torr $)$ after a single laser pulse $\left(1.6 \times 10^{8} \mathrm{~W} / \mathrm{cm}^{2}\right)$ with no applied field. (b) Time profiles showing the number of TFE droplets at $\left(S_{\max }=1.819, T_{\max }=278.6 \mathrm{~K}, P=675.0\right.$ Torr $)$ after a single laser pulse $\left(1.6 \times 10^{8} \mathrm{~W} / \mathrm{cm}^{2}\right)$ under different fields applied between the top and bottom plates of the DCC. Inset shows the total number of droplets as a function of applied electric field. (c) Time profiles showing the number of TFE droplets $\left(S_{\max }=2.39, \quad T_{\max }=269.0 \mathrm{~K}\right.$, and $P$ $=644.2$ Torr $)$ after a single laser pulse $\left(1.6 \times 10^{8} \mathrm{~W} / \mathrm{cm}^{2}\right)$ using negative and positive fields applied to the top plate of the DCC thus attracting positively and negatively charged particles, respectively, to the nucleation zone. (d) Total number of TFE droplets as a function of the magnitude and sign (on the top plate) of the applied fields $\left(S_{\max }=2.39, T_{\max }=269.0 \mathrm{~K}\right.$, and $P=644.2$ Torr).

The inset in Fig. 6(b) clearly shows that the total number of droplets decreases as the applied field increases. This behavior can be attributed to the number of the charged nanoparticles reaching the nucleation zone by drift velocities that increase by increasing the electric field (the drift velocity of the charged particle $\nu_{i}$ is given by $\nu_{i}=\mu_{i} E$, where $\mu_{i}$ is the ion mobility which depends on the size of the ion). In the absence of an applied field, the motion of the nanoparticles is described in terms of diffusion, and the measured nucleation profile reflects the longer time of diffusion as compared to the drift velocity of the charged particles.

The number of droplets forming on the positively charged (applying the negative field on the top plate) and negatively charged (applying the positive field on the top plate) nanoparticles appear to be similar at lower applied fields. However, at higher fields Fig. 6(c) shows that the number of droplets forming on the negatively charged particles is more than the number formed on the positively charged particles. This effect is also observed in the total number of droplets integrated over the nucleation time profile as shown in Fig. 6(d). The number of droplets decreases by similar amounts for the positively and negatively charged particles in the presence of small applied fields. This is due to the removal of ions from the nucleation zone before condensing the supersaturated vapor. This result suggests that both the positive and negative particles have almost the same ability to induce the condensation of TFE vapor. Similar results have been observed by Chen et al. ${ }^{17,18}$ who found that the condensation of supersaturated vapors on singly positive/ negative charged particles had no obvious sign preference. However, Fig. 6(d) shows that at higher applied fields the number of droplets formed on the negatively charged particles appear to be higher than that formed on the positively charged particles (the decrease in the number of droplets by applying positive and negative fields is not symmetric). This could be explained by the low mobility of the negatively charged particles. In this case, the increase in the number of droplets would be due to the longer residence time of the negatively charged particles in the nucleation zone as compared to the more mobile positively charged particles. Therefore, in the current system dealing with the condensation of supersaturated TFE vapor on charged Mg nanoparticles, the sign effect appears to very small or negligible. It should be noted that the situation could be very different in the case of molecular ions where the sign preference could arise from different structures of the positive and negative ions as well as different interactions with the condensing vapor molecules. ${ }^{23,31}$ Previous theoretical studies have observed significant sign effect when molecular-sized charged particles were considered. ${ }^{32,33}$ The current results appear to suggest that the sign effect is smeared out if the charged particle is much larger than the size of the molecules of the condensing vapor. It is clear that definitive answers regarding the sign preference in ion nucleation is still lacking.

\section{SUMMARY AND CONCLUSIONS}

In conclusion, condensation of supersaturated TFE vapor on $\mathrm{Mg}$ nanoparticles generated by laser vaporization has been studied at supersaturations significantly lower than those required for homogeneous nucleation. The number of nanoparticles that can be activated increases with increasing the vapor supersaturation. The carrier gas pressure affects the expansion of the laser plume and the number as well as the size of nanoparticles that act as condensation nuclei. The small difference observed in the number of droplets formed on positively and negatively charged nanoparticles is attributed to the difference in the mobilities of these nanoparticles. Therefore, no significant charge preference is observed for the condensation of TFE vapor on the Mg nanoparticles.

\section{ACKNOWLEDGMENTS}

Acknowledgment is made to the Donors of the Petroleum Research Fund, administered by the American Chemical Society (PRF No. 37582-AC6) and to NASA (NNG04GH45G) for the support of this research.

\footnotetext{
${ }^{1}$ D. Kashchiev, Nucleation: Basic Theory with Applications (ButterworthHeinemann, Oxford, 2000).

${ }^{2}$ L. Laakso, T. Anttila, K. E. J. Lehtinen, P. P. Aalto, M. Kulmala, U. Horrak, J. Paatero, M. Hanke, and F. Arnold, Atmos. Chem. Phys. 4, 2353 (2004).

${ }^{3}$ S. H. Lee, J. M. Reeves, J. C. Wilson, D. E. Hunton, A. A. Viggiano, T. M. Miller, J. O. Ballenthin, and L. R. Lait, Science 301, 1886 (2003).

${ }^{4}$ R. G. Harrison and K. S. Carslaw, Rev. Geophys. 41, 1012 (2003).

${ }^{5}$ M. S. Modgil, S. Kumar, S. N. Tripathi, and E. R. Lovejoy, J. Geophys. Res. 110, D10205 (2005).

${ }^{6}$ S. Wihelm, S. Eichkorn, D. Wiedner, L. Pirjola, and F. Arnold, Atmos. Environ. 38, 1735 (2004).

${ }^{7}$ F. Yu and R. P. Turco, J. Geophys. Res. 103, 25915 (1998).

${ }^{8}$ K. S. Carslaw, R. G. Harrison, and J. Kirkby, Science 298, 1732 (2002).
} 
${ }^{9}$ M. Kulmala, Science 302, 1000 (2003).

${ }^{10}$ M. Adachi, S. Tsukui, and K. Okuyama, J. Nanopart. Res. 5, 31 (2003).

${ }^{11}$ C. T. R. Wilson, Philos. Trans. R. Soc. London, Ser. A 189, 265 (1897); 193, 289 (1899).

${ }^{12}$ Nobel Lectures, Physics 1922-1941 (Elsevier, Amsterdam, 1965).

${ }^{13}$ M. Kulmala, H. Vehkamaki, T. Petaja, M. Dal Maso, A. Lauri, V. M. Kerminen, W. Birmili, and P. J. McMurry, J. Aerosol Sci. 35, 143 (2004)

${ }^{14}$ V. Y. Smorodin and P. K. Hopke, J. Phys. Chem. B 108, 9147 (2004).

${ }^{15}$ R. Mavliev, P. K. Hopke, H. C. Wang, and D.-W. Lee, Aerosol Sci. Technol. 35, 586 (2001).

${ }^{16}$ R. Mavliev, P. K. Hopke, H. C. Wang, and D. W. Lee, J. Phys. Chem. B 108, 4558 (2004).

${ }^{17}$ C. C. Chen, C. J. Tao, and H. C. Cheng, J. Colloid Interface Sci. 255, 158 (2002).

${ }^{18}$ C. C. Chen and W. T. Tsai, J. Colloid Interface Sci. 246, 270 (2002).

${ }^{19}$ M. Rusyniak, V. Abdelsayed, J. Campbell, and M. S. El-Shall, J. Phys. Chem. B 105, 11866 (2001).

${ }^{20}$ C. H. Hung, M. J. Krasnopoler, and J. L. Katz, J. Chem. Phys. 90, 1856 (1989).

${ }^{21}$ F. C. Wen, T. McLaughlin, and J. L. Katz, Science 200, 769 (1978).
${ }^{22}$ J. L. Katz, J. A. Fisk, and V. M. Chakarov, J. Chem. Phys. 101, 2319 (1994).

${ }^{23}$ D. Kane, G. M. Daly, and M. S. El-Shall, J. Phys. Chem. 99, 7867 (1995).

${ }^{24}$ R. Caldwell, D. Wright, and M. S. El-Shall, Z. Phys. D: At., Mol. Clusters 26, 189 (1993).

${ }^{25}$ M. S. El-Shall and S. Li, Adv. Met. Semicond. Clusters 4, 115 (1998).

${ }^{26}$ T. Rosenorn, G. Kiss, and M. Bilde, Atmos. Environ. 40, 1794 (2006).

${ }^{27}$ T. Seto, K. Okuyama, L. De Juan, and J. Fernandez de la Mora, J. Chem. Phys. 107, 1576 (1997).

${ }^{28}$ H. Furusawa, T. Sakka, and Y. H. Ogata, J. Appl. Phys. 96, 975 (2004).

${ }^{29}$ L. V. Zhigilei, P. B. S. Kodali, and B. J. Garrison, Chem. Phys. Lett. 276, 269 (1997).

${ }^{30}$ D. Kane, S. P. Fisenko, and M. S. El-Shall, Chem. Phys. Lett. 277, 6 (1997).

${ }^{31}$ S. P. Fisenko, D. B. Kane, and M. S. El-Shall, J. Chem. Phys. 123, 104704 (2005).

${ }^{32}$ I. Kusaka, Z. G. Wang, and J. H. Seinfeld, J. Chem. Phys. 102, 913 (1995).

${ }^{33}$ K. J. Oh, G. T. Gao, and X. C. Zeng, Phys. Rev. Lett. 86, 5080 (2001). 